\title{
Tinea manuum misdiagnosed as psoriasis vulgaris: A case of tinea incognito
}

\author{
Funda Tamer', Mehmet Eren Yuksel ${ }^{2}$
}

\author{
${ }^{1}$ Department of Dermatology, Medical Park Hospital, Ankara, Turkey, ${ }^{2}$ Department of General Surgery, Devrek State \\ Hospital, Zonguldak, Turkey
}

Corresponding author: Dr. Funda Tamer, E-mail: fundatmr@yahoo.com

\begin{abstract}
Tinea incognito is a dermatophyte infection with altered clinical appearance which is usually caused by the use of immunosuppressive agents such as topical corticosteroids. Hereby, we present a 59-year-old Caucasian male patient with tinea manuum on the dorsum of his left hand. The lesion was formerly misdiagnosed as psoriasis vulgaris and treated with topical corticosteroids. However, the symptoms were worsened. Moreover, new papules and pustules appeared within the lesion. The past medical history was remarkable for psoriasis vulgaris and he had an erythematous and squamous plaque on his lower back resembling psoriasis vulgaris. In order to reach a definitive diagnosis, the skin lesion on the dorsum of the patient's left hand was examined by light microscopy after the application of $10 \%$ potassium hydroxide solution. Detection of septate hyphae confirmed dermatophytosis. The lesion was completely healed with oral terbinafine $250 \mathrm{mg}$ daily for four weeks. Dermatophyte infections in early stages may be misdiagnosed as psoriasis vulgaris and thus, prolonged use of corticosteroids can lead to tinea incognito. Therefore, cutaneous lesions unresponsive to topical corticosteroid treatment should be evaluated with microscopic examination and fungal culture to confirm a suspected dermatophyte infection. Past medical history can provide useful information but a complete dermatological examination should be performed before the final diagnosis is made.
\end{abstract}

Key words: Tinea manuum; Tinea incognito; Psoriasis vulgaris

\section{INTRODUCTION}

Tinea manuum is a fungal infection of the hands caused by dermatophytes. It can present with erythema and minimal scale on the dorsum of the hand. However, chronic tinea manuum lesions located on the palmar region may be scaly and hyperkeratotic. Tinea manuum usually affects the dominant hand but it can also occur bilaterally. The term 'tinea incognito' is used to describe a dermatophyte infection with altered morphology. Tinea incognito usually occurs after inappropriate treatment with topical steroids. Wood's lamp examination, mycological culture, direct microscopy and molecular tehniques like polymerase chain reaction are the methods to reach a definitive diagnosis [1]. Dermatophyte infections can be managed with antifungal and keratolytic agents [2].

\section{CASE REPORT}

A 59-year-old Caucasian male presented with an itchy rash on his left hand for further clinical evaluation. The chief complaint of the patient was pruritus. The lesion on the left hand of the patient was formerly misdiagnosed as psoriasis vulgaris. Therefore, he was treated with topical corticosteroids. Nevertheless, the symptoms were not relieved by the application of topical corticosteroids. In addition, the patient complained of gradual enlargement of the lesion in the last two months. The past medical history was remarkable for diabetes mellitus and psoriasis vulgaris for the past three years. However, he wasn't taking any medications regularly. He admitted that he could manage his blood glucose levels with diet. He was put on topical calcipotriol and clobetasol propionate to treat psoriasis vulgaris. The family history was

\footnotetext{
How to cite this article: Tamer F, Yuksel ME. Tinea manuum misdiagnosed as psoriasis vulgaris: A case of tinea incognito. Our Dermatol Online. 2017;8(1):60-62. Submission: 16.03.2016; Acceptance: 24.08.2016 DOI: 10.7241 /ourd.20171.16
} 
unremarkable. The physical examination showed an erythematous patch with a slightly raised edge on the dorsum of the left hand. There were erythematous, skin colored papules and pustules within this lesion. He also had hyperkeratotic plaques and fissures on both palms (Figs. 1 and 2). In addition, we noticed an erythematous and squamous plaque resembling psoriasis vulgaris on his lower back.

Laboratory tests including blood count and chemistry panel such as urea, glomerular filtration rate, creatinine, alanine amino transferase, aspartate amino transferase, total bilirubin, direct bilirubin were all in the normal ranges. But gamma glutamyl transferase was $56 \mathrm{U} / \mathrm{L}$ (normal range 5-40).

We scraped active edge of the lesion on the left hand using a scalpel and we applied $10 \%$ potassium hydroxide $(\mathrm{KOH})$ solution to the sample. $\mathrm{KOH}$ preparation was examined microscopically and branched hyphae of the fungus were detected. The patient was treated with oral

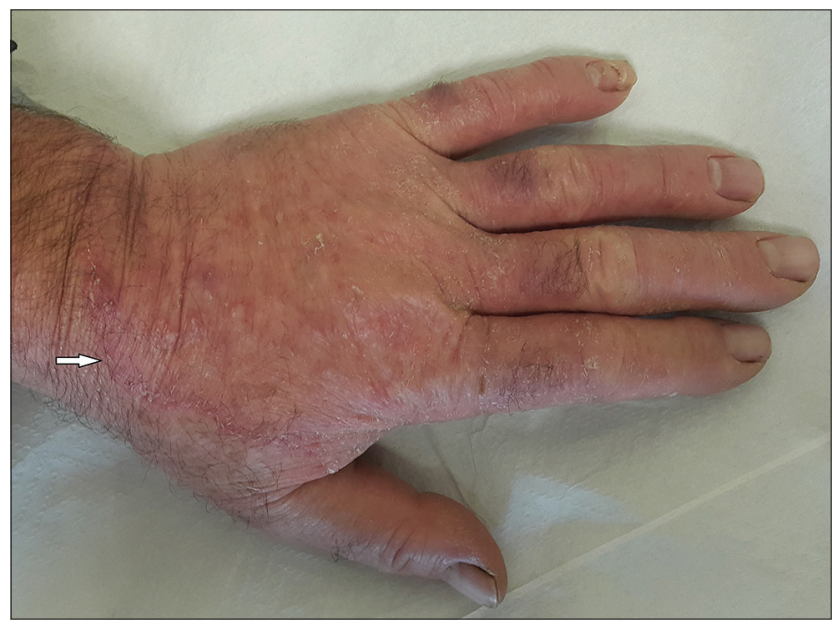

Figure 1: Tinea incognito: Red papules and pustules on erythematous patch with a well defined border on the lateral side of the left hand (white arrow)

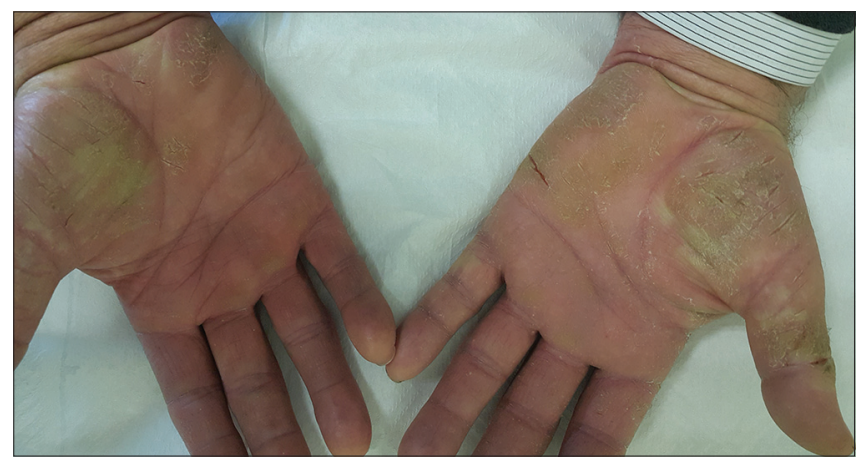

Figure 2: Hyperkeratotic plaques and fissures on the palms terbinafine $250 \mathrm{mg}$ once daily and topical sertaconazole nitrate twice daily. The lesion on the dorsum of the left hand disappeared completely within four weeks.

\section{DISCUSSION}

Tinea manuum is a dermatophyte infection of the palms, interdigital spaces and dorsal aspect of the hands. Fingernails may also be involved. Tinea manuum typically presents with diffuse scaling and hyperkeratosis of the palms and fingers. In addition, annular lesions with erythematous scaly borders may appear on the dorsum of the hand. Exfoliative, vesicular and papular lesions are other clinical manifestations of the tinea manuum. Psoriasis vulgaris, dermatitis, dermatophytid reaction, keratoderma and dyshidrosis should be considered in the differential diagnosis of tinea manuum [3]

The term tinea incognito describes dermatophytic infections with atypical appearance due to topical and systemic immunosuppressants such as steroids and immunomodulators [4]. The lesions are less scaly but more pustular, pruritic, widespread and erythematous than common dermatophytosis. The active margins may be lost [5]. In addition to topical immunosuppressive therapy, virulence of pathogen, individual and environmental factors such as unsanitary conditions may play role in atypical presentation [4] Tinea incognito can mimic other skin disorders such as neurodermatitis, rosacea, lupus erythematosus, contact dermatitis impetigo, lichen planus, psoriasis vulgaris, erythema migrans and drug eruptions. Systemic antifungal agents are usually indicated in patients with tinea incognito [6].

The diagnosis of cutaneous fungal infections may be difficult and tinea manuum can be easily misdiagnosed as psoriasis vulgaris. In our case, the patient's past medical history of psoriasis vulgaris caused a misdiagnosis and delay in the treatment. However, exacerbation of the lesions during treatment with corticosteroids and active border of the lesions led us to reevaluate the initial diagnosis.

\section{CONCLUSION}

In conclusion, dermatophyte infections may present with a variety of unusual clinical features. Dermatophytosis should be kept in mind in differential 
diagnosis of other skin diseases especially when there is no response as expected to topical corticosteroid treatment. A misdiagnosis may lead to chronic and disseminated lesions. Tinea incognito should be treated with systemic antifungal agents and treatment should be continued until the lesions disappear.

\section{Consent}

The examination of the patient was conducted according to the Declaration of Helsinki principles.

\section{REFERENCES}

1. Moriarty B, Hay R, Morris-Jones R. The diagnosis and management of tinea. BMJ. 2012;345:e4380.

2. Drake LA, Dinehart SM, Farmer ER, Goltz RW, Graham GF,
Hardinsky MK, et al. Guidelines of care for superficial mycotic infections of the skin: Tinea corporis, tinea cruris, tinea faciei, tinea manuum and tinea pedis. Guidelines/Outcomes Committee. American Academy of Dermatology. J Am Acad Dermatol. 1996;34:282-6.

3. Sobera JO, Elewski BE. Fungal Diseases. In Bolognia JL, Jorizzo JL, Rapini RP, eds. Dermatology. Spain: Elsewier, 2003; 1178-1179

4. Segal D, Wells MM, Rahalkar A, Joseph M, Mrkobrada M. A case of tinea incognito. Dermatol Online J. 2013;19:18175.

5. Arenas R, Moreno-Coutiño G, Vera L, Welsh O. Tinea incognito. Clin Dermatol. 2010;28:137-9.

6. Zisova LG, Dobrev HP, Tchernev G, Semkova K, Aliman AA, Chorleva KI, et al. Tinea atypica: Report of nine cases. Wien Med Wochenschr. 2013;163:549-5.

Copyright by Funda Tamer, et al. This is an open access article distributed under the terms of the Creative Commons Attribution License, which permits unrestricted use, distribution, and reproduction in any medium, provided the original author and source are credited.

Source of Support: Nil, Conflict of Interest: None declared. 